\title{
Molecular Mechanisms Involved in Inflammatory Cascade: A Review
}

\author{
Article by Jagan Nadipelly \\ Faculty of Medicine-Pharmacology, Texila American University, Guyana, South America \\ E-mail: jaganalwaysright@gmail.com,nadipelly.j@tau.edu
}

\begin{abstract}
Inflammation participates importantly in host defenses against infectious agents and injury, but it also contributes to the pathophysiology of many chronic diseases. Many molecular mechanisms are involved in the process of inflammation such as Prostaglandins, Platelet activating factors, Leukotrienes, Tumor

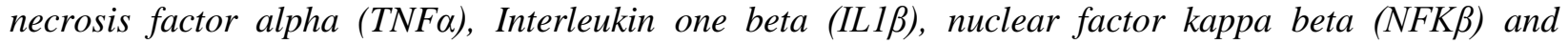
oxidative stress. These inflammatory molecules work together in concert to produce inflammation. The therapeutic targets for resolving inflammation are numerous because the process of inflammation is multifaceted. Inflammatory mediators, free radical activity and oxidative stress have been found to be attractive anti-inflammatory targets. The role of these components must be understood in order to effectively investigate inflammatory mediators as drug targets. This review mainly aims to summarise our current understanding of the molecular basis of inflammation and therapeutic implications to prevent this phenomenal activity.
\end{abstract}

Keywords: Inflammation, Bradykinin, Adenosine triphosphate, Tumor necrosis factor, nitric oxide synthase, Prostaglandins, nuclear factor kappa beta.

\section{Introduction}

The term inflammation is derived from the Latin word - Inflammare, means burn. Any form of injury to the human body can elicit a series of chemical changes in the injured area (Park et al., 2013). Earlier it was believed that inflammation was contemplated as a single disease caused by disturbances of body fluids. According to the modern concept, inflammation is a healthy process resulting from some disturbance or disease (Krishnaraju et al., 2009). The cardinal signs of inflammation are heat, redness, swelling, pain and loss of function (Hsu et al., 2013). Inflammation usually involves a sequence of events which can be categorized under three phases viz. acute transient phase, delayed sub-acute phase and chronic proliferate phase (Vadivu and Lakshmi, 2011). In the first phase, inflammatory exudates develop due to enhanced vascular permeability and leads to local edema (Hong et al., 2009). It is followed by the migration of leukocytes and phagocytes from blood to vascular tissues which are the second phase, in the third phase; tissue degradation is followed by fibrosis (Wakefield and Kumar, 2001).

Inflammation results in the liberation of endogenous mediators like histamine, serotonin, bradykinin, prostaglandins etc. Prostaglandins are ubiquitous substances that indicate and modulate cell and tissue responses involved in inflammation (Payne, 2000). These mediators even in small quantities can elicit pain response.

\section{Mediators of inflammation}

Many endogenous chemical mediators play a significant role in mediating various reactions of the inflammatory process like vascular permeability, vasodilation, chemotaxis, cellular migration and tissue damage. Inflammation is mediated by many inflammatory mediators such as chemokine's hat include tumor necrosis factor (TNF)- $\alpha$, nuclear factor kappa beta (NFK $\beta$ ), interferon (IFN)- $\gamma$, nitric oxide and interleukins, may originate from different types of cells, plasma or from the damaged tissue itself (Hong et al., 2009). Most inflammatory mediators are newly synthesized in the injured tissues or by the migrated immune cells during an inflammatory event (Wakefield and Kumar, 2001). Pathogenic microorganisms 
such as bacteria activate NF-K $\beta$ through receptors found on macrophages via several signalling pathways. The activated macrophages can release TNF- $\alpha$ which is responsible for the up-regulation of the production of other inflammatory mediators that include prostaglandins, nitric oxide and free radicals (Franceschelli et al., 2011; Posadas et al., 2003). The presence of large quantities of free radicals creates conditions that allow the liberation of NFK $\beta$ and this NFK $\beta$ is move into the nucleus to promote transcription of COX-2 and iNOS genes (Zhao et al., 2009). Eventually there is an overproduction of Cyclooxgenase- 2 and inducible nitric oxide synthase, which resulting in inflammation.

Cyclooxygenase (COX) enzymes are membrane bound glycoproteins found in the endoplasmic reticulum (Gacche et al., 2012). Arachidonic acid is converted by cyclooxygenase enzymes to prostaglandin $\mathrm{H} 2$, the precursor molecule for all the eicosanoid molecules that include prostaglandins and thromboxanes (Bai and Zhu, 2008). There are two main isoforms of COX enzymes that differ mostly in their pattern of expression. The isoforms share about $60 \%$ of their overall sequence and the maximum homology in the catalytically-important regions. The major differences between the isoforms are in substrate and inhibitor selectivity, their intracellular locations and the circumstances under which they are expressed. The substrate and inhibitor selectivity's are found in the regions where the sequence differences between the two isoforms are located. COX-1 is constitutively expressed, that is, the enzyme is found under normal physiological conditions, throughout most of the tissues of the body. Whereas, COX-2 expression is induced by pro-inflammatory conditions during an innate immune response, although it is constitutively expressed in the brain and kidneys (Mulabagal et al., 2011). The expression of COX-2 enzymes is the rate limiting step in the production of the prostaglandins that act as proinflammatory mediators. COX-2 has been found to be overexpressed in prostate cancer cells and individuals who took non-steroidal anti-inflammatory drugs were found to have a lower incidence of the disease (Lin et al., 2011). Studies in animal models have shown that pharmacological inhibition or genetic deletions of COX-2 have led to the suppression of tumor growth (Yan et al., 2006). The inhibition of COX-2 could affect the production of downstream inflammatory mediators including prostaglandins (Turini and DuBois, 2002).

\section{Bradykinin}

Bradykinin is a low molecular weight vasoactive kinin, formed by nine amino acids. It has short plasma half-life and executes its action through bradykinin receptor, called B1 (B1R) and B2 (B2R) receptors. Usually bradykinin induce inflammation induces inflammation by releasing pro-inflammatory cytokines and/or increasing vascular patency. Bradykinin B2 receptor is constitutively expressed by peripheral and central tissues, while B1 receptor is induced and regulated according to the evolution of infection, inflammation or trauma. In the nervous system, for example, B2 receptor is found, in normal situations, in different types of cells, including sensory neurons and microglial cells, while B1 receptor is sensitized in microglial cells, astrocytes and neurons after tissue injury. B2 receptors of bradykinin alone contributes for the acute inflammatory and nociceptive response phase. (Dawes et al., 2013). Bradykinins induced inflammation by perpetuating cytokines and chemokines inflammatory cascade, as well as to stimulate histamin release by mast cells. (Moalem et al., 2006).

\section{Adenosine triphosphate}

The complex panel of action of adenosine triphosphate (ATP) and its involvement in several pathophysiologic mechanisms is just recently emerging and generating scientific interest. It executes it actions through the activation of inotropic ( $\mathrm{P} 2 \mathrm{X}$, ion channel) and metabotropic (G protein-bound $\mathrm{P} 2 \mathrm{X}$ ) membrane receptors. In fact, ATP is a multifunctional molecule involved in signal transduction by cells, when it released in the extracellular space after cell destruction, induce inflammation by producing inflammatory mediators such as BDNF and glial pro-inflammatory cytokines. (Austin et al., 2013).

The activation of $\mathrm{P} 2 \mathrm{X} 7$ receptors in immune cells (mast cells, macrophages, T lymphocytes and microglia) induces fast activation and secretion of interleukin $1 \beta$ (IL-1 $\beta$ ), which resulting in acute inflammation. (Honore et al., 2009). P2Y12 receptors also activate microglia and contribute to 
inflammation. Inhibition of these receptors by P2X12 antagonists or genetic deletion of gene P2ry12 relieves inflammatory pain in many animal models. So, purinergic system is a promising set of therapeutic targets to control inflammatory processes.

\section{Tumor necrosis factor}

Tumor necrosis factor (TNF) is an important pro inflammatory cytokine and acts by two receptors: constantly expressed TNFR1 and inducible TNFR2. TNF starts several signalling pathways: activates nuclear factor (NF)-KB, which induces transcription of pro-inflammatory cytokine coded genes, thus triggering an inflammatory cascade. (Wei et al., 2007) TNF increases vascular patency in hairless skin increasing leucocytes infiltration and consequently inflammatory response. These effects support the theory which suggests that TNFs are peripheral sensitizers. Nervous injuries also trigger increased TNF in spinal cord dorsal part, which increases NAV 1.3 and NAV 1.8 sodium channels expression, as well as increases post-synaptic excitatory chain by means of AMPA and NMDA receptors, both susceptible to inflammatory reaction. (Whitehead et al., 2010). The interference of TNF signalling using neutralizing antibodies or antagonists of their receptors may attenuate various types of inflammation (Austin et al., 2013). In the last decade, treatment with anti-TNF (infliximab, etanercept) has positively transformed the treatment of severe inflammatory diseases, such as rheumatoid arthritis, inflammatory bowel diseases and psoriasis.

\section{Prostaglandins}

Prostaglandins are bioactive derivatives of unsaturated fatty acids that are involved in the mediation of clotting, anti-clotting, pain, fever, inflammation and anti-inflammation (Payne, 2000). Prostaglandins are produced by the isomerization of prostaglandin $\mathrm{H} 2$ by respective prostaglandin synthases (Figure 1) and modulate cellular and tissue responses involved in inflammation. The process begins when phospholipase A2 (PA2) is activated to break down membrane phospholipids to arachidonic acid. Arachidonic acid is the first precursor molecule in the production of the prostaglandins. The COX-1 and - 2 act on arachidonic acid in two steps to produce prostaglandin $\mathrm{H} 2$. The first step involves the cyclooxygenase of arachidonic acid which produces an arachidonic acid radical which is then acted on the by the peroxidase segment of the enzyme to produce prostaglandin $\mathrm{H} 2$ (PGH2). PGH2 then diffuses to the different prostaglandin $\mathrm{H} 2$ isomerizes in the cell to produce the functional terminal prostaglandins. The terminal prostaglandins produced have various functions. However, there are three prostaglandins that are known to be proinflammatory, prostaglandin D2 (PGD2), prostaglandin E2 (PGE2) and prostaglandin F2 $\alpha$ (PGF2 $\alpha$ ) (Ricciotti and Fitzgerald, 2011). Prostaglandin D2 and prostaglandin F are responsible for vasodilation and permeability of vessels. However, prostaglandin E2 (PGE2) is particularly important due to the roles it plays in inflammation. Prostaglandin E2 is a product of microsomal prostaglandin E synthase-2, an enzyme that is mostly expressed during an inflammatory event. 


\section{Membrane Phospholipids}

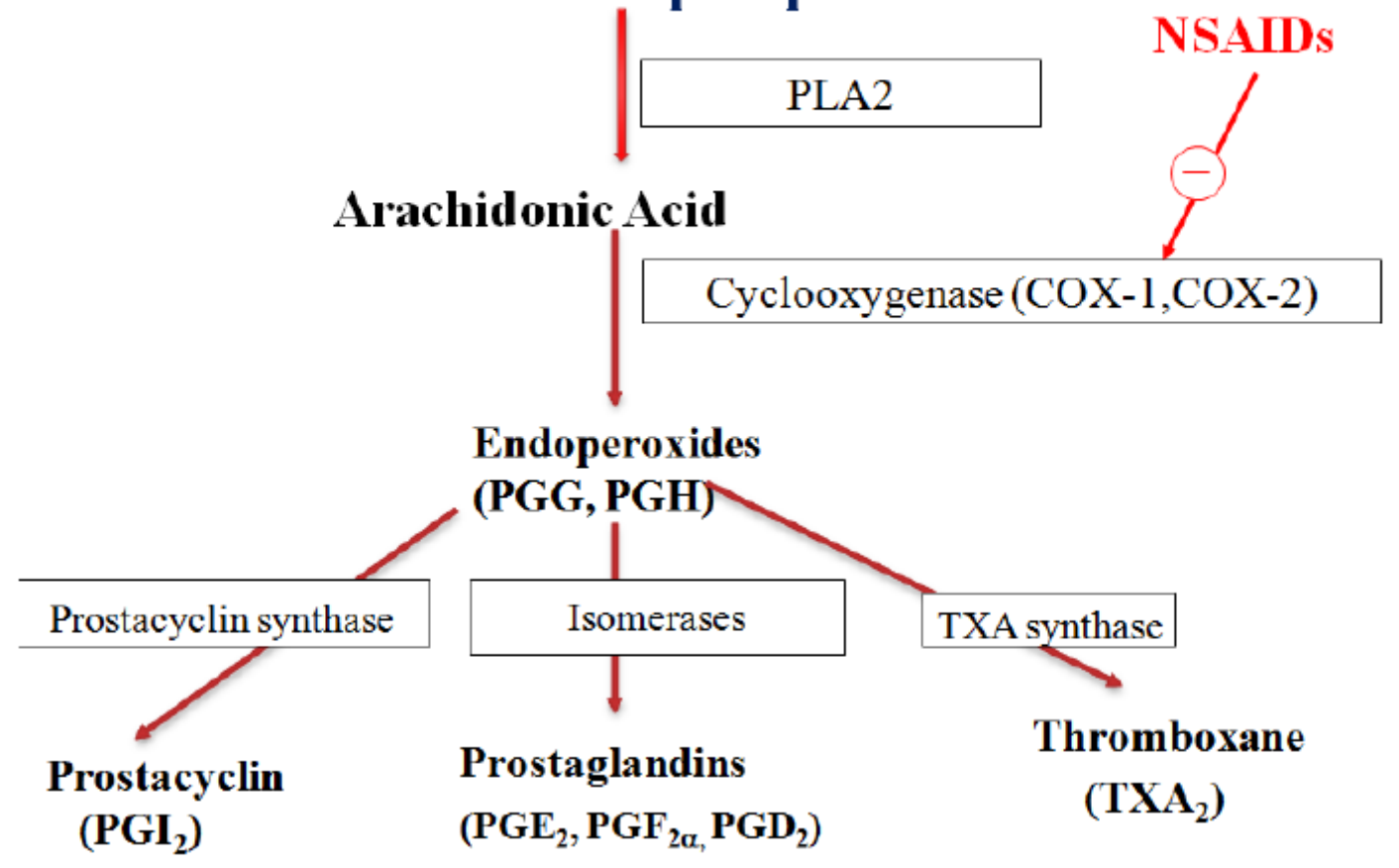

Figure1. Pathway of synthesis of prostaglandins

PGE2 has pleiotropic roles in inflammation that are known to be vasodilation, stimulation of the central nervous system for the conduction of pain and its pyrogenetic effect on the hypothalamus. PGE2 is involved in the activation of $\mathrm{T}$ cells that recruit more immune cells to the site of inflammation during an infection or autoimmune response (Takanishi and Rosenberg, 2013). The COX-2 induction of expression by PGE2 leads to a positive feedback mechanism that allows more PGE2 to be produced by the macrophages and the effector cells (Hernandez et al., 2011). If the induction and production cycle is not properly regulated and or terminated the large quantities of PGE2 produced can exacerbate the inflammatory response. PGE2 and its metabolite PGF2 $\alpha$ have another physiological role in the gonads as well that plays a part in disease, particularly in females. The prostaglandins were first isolated from human seminal vesicles, scientists believed the lipid derived molecules were localized in the prostate, hence the name prostaglandin (Zhuang et al., 2003). The prostaglandins were later discovered in other locations and organs such as the uterus. The prostaglandins, PGE2 and PGF $\alpha$, were found to play an important role in the uterus. PGE2 and PGF $\alpha$ are highly expressed during pregnancy, labour and the menstrual cycle (Sales and Jabbour, 2003). PGE2 is responsible for muscle contraction and relaxation to allow menstrual flow. The over-expression of the bioactive fatty acids can lead to a condition known as dysmenorrhea. Dysmenorrhea is a pain disorder of the uterus that can result from the overproduction of PGE2 and PGF $\alpha$ within the uterine muscles (Arif Zaidi et al., 2012). As mentioned previously PGE2 mediates pain by activating the neurons that transmit pain signals and overproduction of the prostaglandins leads to uterine muscle cramping due to oxygen deprivation. The role of PGE2 in diseases such as cancer and dysmenorrhea, therefore, should not be underestimated. PGE2 has been shown to modulate the production of other inflammatory mediators. Milano et al., (1995) speculated that PGE2 production influences the production of the free radical, nitric oxide (NO) in a murine macrophage cell line. 


\section{Inducible nitric oxide synthase}

Nitric oxide (NO) is act as a neurotransmitter, involved in vascular homeostasis and maintain host defense. There are three isoforms of the nitric oxide synthase, which include endothelial NOS (eNOS) and neuronal NOS (nNOS), constitutively expressed and responsible for low levels of NO production. On the other hand, inducible NOS (iNOS), is calcium-independent, not expressed under normal physiological conditions, but can be induced by an immune reaction to injury. Nitric oxide activates protein kinase pathways such as the mitogen activating protein kinase (MAPK) involved in inflammation making it a key enzyme in the inflammatory response (Lee et al., 2011). MAPK are involved in the cascade responsible for induction of expression of inflammatory cytokines. NO from eNOS and nNOS is released from the cell that produced it and diffuses into its target cells through the lipid membranes where the radical binds to guanylate cyclase. The guanylate cyclase produces large quantities of the messenger cyclic guanidine monophosphate (cGMP) that effects muscle contraction and inhibits platelet aggregation (Jerca et al., 2002). NO produced by iNOS is in large quantities that can be cytotoxic to any invading pathogens while acting on effector cells to inhibit platelet aggregation and effect muscle contraction. Unregulated production of NO by iNOS during or after an inflammatory event can lead to an excess of the signalling radical circulating the site of injury (Cheenpracha et al., 2010). Excessive production of the radical NO has been associated with diseases such as rheumatoid arthritis, diabetes and hypertension. The enzyme iNOS, produces up to 1000 times more NO than its constitutive isoforms, eNOS and nNOS, for a relatively extended period of time (Park et al., 2013). The increased NO production by iNOS could relate to other mediators such as prostaglandin whose overproduction are associated with development of disease. A link between the production of NO and the prostaglandins has been established as well that shows that NO is involved in increasing COX-2 activity. NO has been shown to nitrosylate the cysteine residues of COX-2 thereby enhancing its catalytic activity (Kim et al., 2005). The enhanced COX-2 activity could increase the amount of PGH2 available for the production of PGE2. Therefore, the improper regulation of the expression of iNOS and NO production can create the chronic microenvironments that are ideal for disease pathogenesis and progression of inflammatory conditions. Aside from being an effector molecule in inflammation, NO is a highly reactive free radical. The radical state means the molecule has a lone electron that allows it to react with other molecules in order to reach the octet state to be stable. NO radical is usually short-lived as it is converted to the more stable form of nitrites by nitric oxide reductase in the cell. However, if the turnover of the enzyme is lower than the generation of the radical it can lead to protein, lipid and deoxyribose nucleic acid (DNA) damage. The damage to the biomolecules is as a result of the abstraction of an electron by the radical. Due to this activity, NO is classified as part of a group of non-biological molecules that are generated by the body during cellular respiration and bioprocesses called reactive oxygen species (Halliwell, 2001).

\section{Reactive oxygen species and oxidative stress}

Reactive oxygen species (ROS) are atoms or group of atoms that has one or more unpaired electrons or molecular species that are capable of generating free radicals (Kunwar and Priyadarsini, 2011). Inflammation generates large quantities of reactive oxidative species (ROS) that when overproduced and not eliminated can lead to damage at the site of inflammation which can lead to recruitment of more immune cells. ROS come from both normal metabolism and external sources (Lai et al., 2011). The oxidants are produced by the body for physiological function, such as NO which is required for neurotransmission or as by-products of cellular processes such as superoxide produced by nicotinamide adenosine diphosphate (NADP) oxidase during the electron transport chain (Hamilton et al., 2004). Free radicals can be generated during an inflammatory response called the respiratory burst. The respiratory burst is when immune cells such as phagocytes and macrophages release noxious molecules such NO, hypochlorous and hydroxyl radicals in order to kill harmful bacteria and viruses (Biller- Takahashi et al., 2013). The free radical species can come from the external environment as well in form of smoke, ultraviolet radiation, pesticides and air-pollution (Masoko and Eloff, 2007). ROS produced are usually 
quenched by the enzymatic and non-enzymatic reactions in the body. For instance, the superoxide and hydrogen peroxide radicals are broken down to water and oxygen by the enzymatic actions of superoxide dismutase and catalase respectively. Glutathione $(\mathrm{GSH})$ and selenium are some of the non-enzymatic mechanisms that conjugate to the radicals or quench ROS by donating an electron. However, these mechanisms can be overwhelmed or ineffective, particularly in chronic inflammatory conditions where there the free radicals can be perpetually generated (Patel et al., 2010). High levels of oxidation may result in oxidative stress which is an imbalance between production of ROS and the capacity of the normal detoxification systems to remove the radicals (Bulbul et al., 2011). Oxidative stress is usually harmful to the cells due to the high reactivity of the radical species that cause it. ROS and reactive nitrogen species are free radicals, which mean the molecules have a lone electron in the outer shell. The lone electron makes radicals highly reactive as they seek stability by abstracting an electron from nearby molecules (Das Sarma et al., 2010). The reactivity and instability of ROS give them ability to react with each other as they seek stability but can instead produce even more potent free radical species. An example of the result of two radicals reacting is peroxynitrite which is produced by the combination of superoxide and nitric oxide as shown in Figure 2. Peroxynitrite is highly reactive with lipid and protein molecules which can lead to the formation of lipid peroxides that can chain react to produce more ROS (Sakat et al., 2010). Other ROS and reactive nitrogen species that are inclusive of the hydroxyl, hypochlorous, superoxide or nitric oxide radicals are able to abstract electrons from DNA, lipids and proteins. The removal of the electrons from functional biomolecules can result in loss of structure which can disrupt the proper function of the cells. ROS can facilitate the formation of DNA adducts and protein cross linkages especially in transmembrane proteins as the biomolecules seek stability themselves. In conditions of oxidative stress, the probability of adducts, cross linkages and lipid peroxides forming is increased exponentially and could be the cause of disease (Khansari et al., 2009).

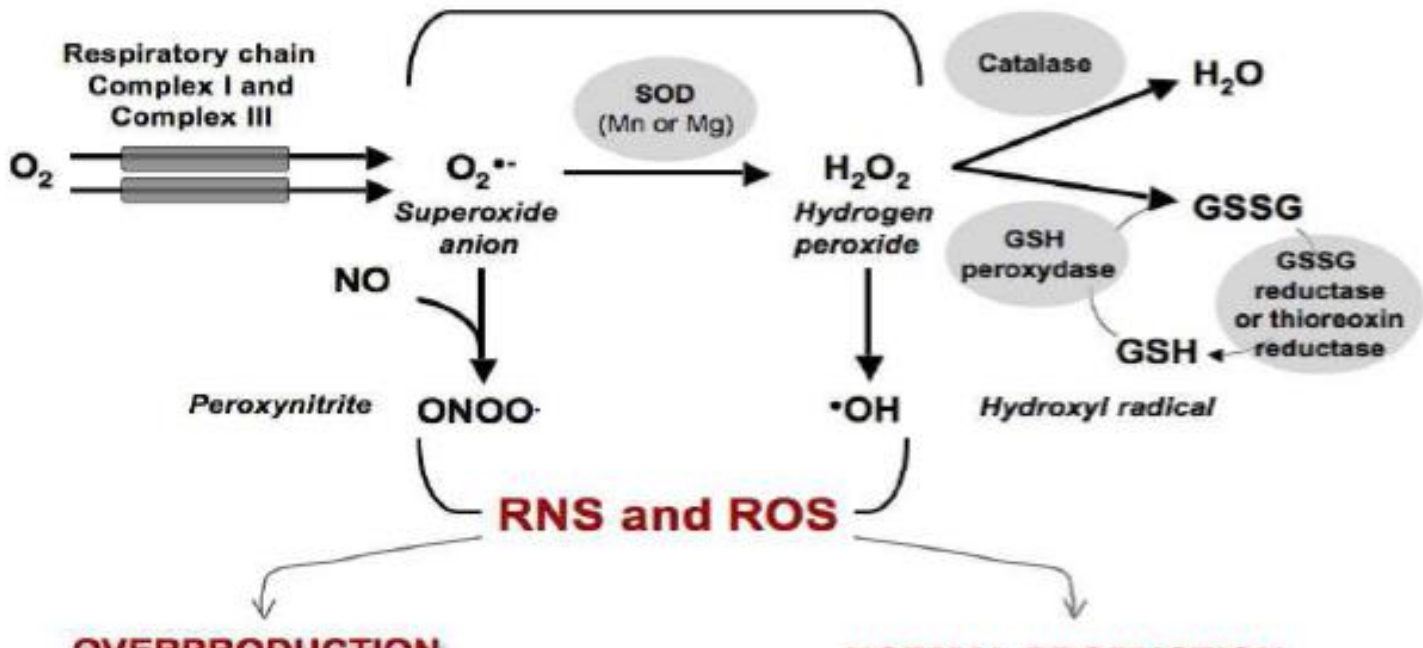

OVERPRODUCTION

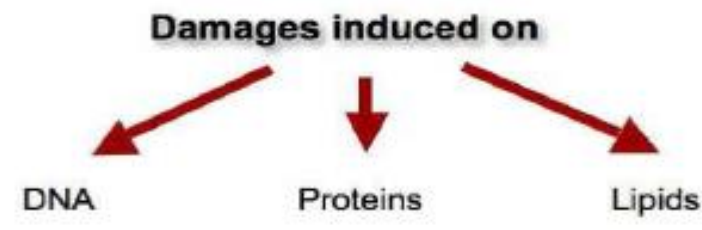

NORMAL PRODUCTION

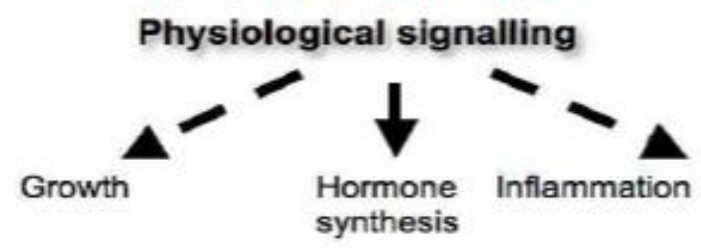

Figure 2. Reactive oxygen species and their role in the induction of disease

Source: Rang and dale's pharmacology, $8^{\text {th }}$ edition.

Oxidative stress is considered one of the major factors in the induction of many chronic and degenerative diseases such as cancer, Alzheimer's and cardiovascular diseases. The excess ROS produced 
that cannot be neutralized create a microenvironment that facilitates normal cells to transform and transformed cells to metastasise to other parts of the body (Pala and Gurkan, 2008). Free radicals have been implicated in the lesions which could be responsible for the memory loss associated with Alzheimer's disease (Das Sarma et al., 2010). The role ROS play in atherosclerosis is that the interaction of the radicals with the proteins and lipids that make the endothelia of the vessels allows plaques to form. The plaques attract immune cells and platelets that attempt to repair the lesion which could result in obstruction of the blood vessels (Lobo et al., 2010).

\section{Inhibition of inflammatory mediators}

Inflammation, acute or chronic, could be resolved by inhibiting inflammatory mediators, quenching of radicals and prevention of oxidation stress. Inhibition can be reversible or irreversible depending on the pathway, molecule or process being targeted. Inhibition can target expression or production of the mediator or its activity directly. In order to inhibit inflammation at the level of expression of the mediators, NFK $\beta$ would be an attractive target since it is responsible for the up-regulation of the expression of the inducible enzyme systems. Preventing or down-regulating NFK $\beta$ translocation to the nucleus would result in less COX-2 and iNOS expression which could reduce or eliminate the inflammatory response (Lee et al., 2011). Modulating expression of the inducible enzyme systems by inhibiting NFK $\beta$ translocation would probably down-regulate inflammation in chronic conditions such as rheumatoid arthritis and atherosclerosis. The potential of inhibiting the translocation of NFK $\beta$ for antiinflammatory effect would probably prevent the adverse effects associated with inhibiting constitutive forms of the enzymes. Wu et al., (2013) showed that the Gynura bicolor extract inhibited the dissociation of NFK $\beta$ from its inhibitor IK $\beta$. The inhibition of translocation of NFK $\beta$ led to a lower expression of iNOS and COX-2. However, despite the potential of targeting translocation of NFK $\beta$ as an antiinflammatory mechanism, inhibiting the process might have far-reaching consequences due to the ubiquitous nature of the nuclear factor. Although, targeting inflammatory mediators has been a means of therapy for inflammatory conditions, inhibiting the cyclooxygenases is the current medical intervention in inflammatory conditions. Most inflammatory drugs inhibit both isoforms despite COX-2 being the enzyme that is directly involved in inflammatory events (Simmons et al., 2004). COX-1 being the constitutive form is essential for normal physiological function as it is responsible for the production of prostaglandins, thromboxanes and leukotrienes that are not pro-inflammatory. Thromboxanes and Prostacyclins are important to physiology even when there is no inflammatory response. For example, thromboxanes are involved in the clotting of blood and Prostacyclins are anti-inflammatory, therefore, inhibition of COX-1 might actually retard resolution of injury (Rao, 2010). On the other hand, COX-2 inhibition would be desirable in the therapy of inflammation and to prevent the aetiology of disease due to chronic inflammation. COX- 2 has been found in large concentrations at tumor sites in gastric, pancreatic and oesophageal cancers. The selective inhibition of COX-2 activity would be ideal as it is induced when the inflammatory response is activated. As the conversion of arachidonic acid by COX-2 is considered the rate limiting step in the production of prostaglandins, inhibition of the enzyme would have an impact on the overall inflammatory response. Inhibiting the enzyme has an influence on the production of prostaglandin E2, which is heavily implicated in development of cancer (Marnett, 2009). Regulation of prostaglandin production in the tissues is important for both maintenance of function and prevention of disease. PGE2 is implicated in the aetiology of cancer by creating a suitable microenvironment for the development of disease. The prostaglandin has been found to be overexpressed at tumor sites (Lawlor et al., 2010). PGE2 promotes the inhibition of apoptosis. Metastasis of transformed cells and cell proliferation are influenced by the prostaglandin as well. PGE2 induces angiogenesis, the development of new blood vessels that carry nutrients and oxygen to the neoplasm. All these factors are hallmarks of carcinogenesis and account for why PGE2 is implicated in the aetiology of diseases such as cancer (Lam et al., 2004). Therefore, inhibition of prostaglandin E2 production would be most beneficial for the prevention of cancer. Microsomal prostaglandin E2 synthase is a possible target for the resolution of 
inflammation. Inhibition of PGE2 could influence the regulation of other mediators in the response. PGE2 has been shown to influence the induction of COX-2 and iNOS expression in immune cells during an inflammatory event (Milano et al., 1995). The inhibition of nitric oxide production might another possible target. iNOS produces larger proportions of NO in a shorter period of time compared to the constitutive forms of the enzyme. NO radical has a dual role in the immune response, first as an inflammatory effector molecule that activates other pathways and then as a free radical species that can be toxic to pathogens. Both roles allow NO to be important in terms of chronic inflammation. A novel anti-inflammatory that would target iNOS specifically would probably significantly reduce occurrence of chronic inflammation and oxidative stress. The ideal target for iNOS inhibition would probably be its expression after activation of the immune system to discontinue the downstream effects of the NO radical. Prevention of oxidative stress might have an anti-inflammatory effect or prevent the commencement of chronic inflammation which may lead to disease. Antioxidants could an effective way to eliminate the free radicals that cause oxidative stress (Kumar et al., 2010). Antioxidants are molecules that are required in small quantities to prevent or delay the oxidation of an oxidisable substance (Charles, 2013). Oxidative stress usually comes about when the endogenous antioxidant systems are overwhelmed by reactive oxygen species and free radical generation within the cell. Therefore, antioxidants from external sources are required to restore the oxidative balance. Exogenous antioxidant compounds are beneficial in biological systems which have insufficient amounts of endogenous antioxidants. The removal of inflammatory signalling molecules such as NO might result in the down-regulation of the inflammatory response. Studies have found that there are interactions between antioxidant agents and inflammatory mediators involved in inflammatory processes (Pegg, 2009). An example of how all the inflammatory mediators and their effects play a role in the aetiology of disease is the development and progression of rheumatoid arthritis. The prevention of oxidative stress or increasing the antioxidant capacity within the cells could result in the alleviation of inflammation, if not its prevention.

\section{Current treatment for inflammation and oxidative stress}

Of all the therapeutic agents in the world, NSAID are the most widely used compounds. However, the available NSAID drugs have reduced efficacy against inflammatory conditions due to adverse effects and relatively high potency. All these effects are observed due to the primary effect of these compounds in inhibiting prostaglandin synthesis. Current treatment of inflammatory diseases involves mainly interrupting the synthesis or action of critical mediators that drive the host's response to injury (Tung et al., 2008). Steroidal anti-inflammatories inhibit basal physiological function such as leukotriene inhibition. The non-steroidal drugs induce gastrointestinal bleeding and improper clotting of blood (Burk et al., 2010). The selective COX-2 inhibitors such as celecoxib and rofecoxib are possible solution for devoid of these characteristics (Kaur et al., 2010). They selectively inhibit the expression and activity of COX-2 could result in the reduction of the inflammatory response without affecting the constitutive COX-1 (Miguel, 2010). However, they induce cardio-, nephro and hepatotoxic effects due to the inhibition of the production of the baseline concentration of COX-2 that is required for normal physiological function (Kaur et al., 2010). Two synthetic COX-2 inhibitors, rofecoxib and valdecoxib, have been withdrawn from the market by the Food and Drug Administration (FDA) due to cardiovascular complications and skin reactions (McDaid et al., 2010). The selective inhibitors developed could play another role of quenching free radicals that attenuate chronic inflammation. In fact, aspirin has been shown to possess antioxidant activity and this has been speculated to another mechanism of action besides the inhibition of COX enzymes. The antioxidant activity of an anti-inflammatory drug could imply that the reverse could be true and an antioxidant supplement could serve a dual role as an anti-inflammatory. Although presently, there are no antioxidants in use clinically for the treatment of inflammation the strong link between oxidative stress and inflammation might imply that antioxidant supplements might play a role in alleviating or preventing inflammatory conditions. Studies have been shown that vitamin E, a known antioxidant, was able to inhibit COX-2 (Akkol al., 2012). Examples of currently available 
antioxidants are the synthetic compounds, butylated hydroxytoluene (BHT) and butylated hydroxyanisole (BHA). The antioxidants are used as food additives and supplements to prevent and or remove oxidative species. However, despite being effective as antioxidants, the two compounds have been found have adverse effects that include carcinogenesis, liver and kidney toxicity. Long term regular long-term use of low dose NSAIDs has been associated with a significant reduction in the incidence of colon cancers by approximately $40 \%$ (Lam et al., 2004). Therefore, the ultimate goal would be to obtain anti-inflammatory agents that are effective with minimal to no adverse effects when administered over a long-term period. The agents would have the dual role of treating inflammation and preventing development of chronic and degenerative diseases. Plants have been considered to be sources of the new anti-inflammatory agents due to their extensive use in folk medicine globally to treat various ailments (Boukhatem et al., 2013).

Currently, clinical trials have not revealed effective treatments to prevent the development of chronic inflammation. NSAIDs, a mainstay for many forms of inflammation, provides only limited relief and does so at the risk of other complications. It is, therefore, important to find alternative treatments. Many of the mechanisms discussed here have been studied as potential avenues of inflammatory treatment, but the clinical trials have produced mixed results. When studying inflammatory mechanisms and developing treatments on the basis of preclinical studies, it is important to keep in mind issues of validity and reliability.

\section{References}

[1].Akkol, Das S., Sarker, SD. and Nahar L. (2012). The treatment of inflammation, pain, and fever using medicinal plants. Advances in Pharmacological Sciences. 2012: 476985.

[2].Arif Zaidi, SM., Khatoon, K and Aslam, KM. (2012). Role of herbal medicine in Ussuruttams (Dysmenorrhoea). Journal of Academic and Industrial Research. 1: 113-117.

[3].Arima, M. and Fukuda, T. (2011). Prostaglandin D2 and TH2 inflammation in the pathogenesis of bronchial asthma. Korean Journal of Internal Medicine. 26: 8-18.

[4].Austin PJ, Moalem-Taylor G. Pathophysiology of neuropathic pain: inflammatory mediators. In Toth C, Moulin DE, (editors). Neuropathic Pain. Cambridge University Press: New York; 2013 ; (7):77-89.

[5].Bai, H. and Zhu, BT. (2008). Strong activation of cyclooxygenase I and II catalytic activity by dietary bioflavonoids. Journal of Lipid Research. 49: 2557-2570.

[6].Biller-Takahashi, JD, Takahashi, LS, Saita, MV, Gimbo RY and Urbinati, EC. (2013). Leukocytes respiratory burst activity as an indicator of innate immunity of pacu Piaractus mesopotamicus. Brazillian Journal of Biology. 73: 425-429.

[7].Boukhatem, MN. Kameli, A., Ferhat, MA, Saidi, F. and Mekarnia M. (2013). Rose geranium essential oil as a source of new and safe anti-inflammatory drugs. Libyan Journal of Medicine. 8: 10.

[8].Bulbul, IJ, Near, L. and Haque M. (2011). Antibacterial, cytotoxic and antioxidant activity of chloroform, nhexane and ethyl acetate extract of plant Coccinia cordifolia. Agriculture and Biology Journal of North America. 2: 713-719.

[9].Burk, DR., Cichacz, ZA., and Daskalova SM. (2010). Aqueous extract of Achillea millefolium L. (Asteraceae) inflorescences suppresses lipopolysaccharide-induced inflammatory responses in RAW 264.7 murine macrophages. Journal of Medicinal Plants Research. 4: 225-234.

[10]. Charles, D. J. (2013). Antioxidant assays. In: Antioxidant Properties of Spices, Herbs and Other Sources. Springer Science + Business Media, New York, 9-38.

[11]. Cheenpracha, S., Park, E., Rostama, B., Pezzuto, JM., and Chang, LC. (2010). Inhibition of nitric oxide (NO) production in lipopolysaccharide (LPS)-activated murine macrophage RAW 264.7 cells by the norsesterterpene peroxide, epimuqubilin A. Marine Drugs. 8: 429-437.

[12]. Chen, ZJ. (2005). Ubiquitin signaling in the NF-K $\beta$ pathway. Nature Cell Biology. 7: 758-765.

[13]. Das Sarma, A., Mallick, AR. and Ghosh AK. (2010). Free radicals and their role in different clinical conditions: An overview. International Journal of Pharma Sciences and Research. 1:

[14]. 185-192. 
Texila International Journal of Basic Medical Science

Volume 2, Issue 1, Jul 2017

[15]. Dawes JM, Anderson DA, Bennett DL, Bevan S, McMahon SB. Inflammatory mediators and modulators of pain. Wall and Melzack's Textbook of Pain. 2013; 6:48-67

[16]. Franceschelli, S., Pesce, M., Vinciguerra, I., Ferrone, A., Riccione, G., Antonia, P., Grilli, A.,

[17]. Felaco, M. and Speranza L. (2011). Licocalchone-C extracted from Glycyrrhiza glabra inhibits Lipopolysaccharide-Interferon- $\gamma$ inflammation by improving antioxidant conditions and regulating inducible nitric oxide synthase expression. Molecules. 16: 5720-5734.

[18]. Gacche, R., Shaikh, R., Pund, M. and Rupesh, Deshmukh. (2012). Cyclooxygenase inhibitory, cytotoxicity and free radical scavenging activities of selected medicinal plants used in Indian traditional medicine. Pharmacognosy Journal. 3: 57-64.

[19]. Halliwell, B. (2001). Free radicals and other reactive species in disease. Encyclopedia of Life Sciences. Nature Publishing Group. 1-7.

[20]. Hamilton, CA., Miller, WH., Al-Benna, S., Brosnan, MJ, Drummond, RD., McBride, MW and Dominiczak, AF. (2004). Strategies to reduce oxidative stress in cardiovascular disease. Clinical Science. 106: 219-234.

[21]. Hernandez, Y., Sotolongo, J. and Fukata, M. (2011). Toll-like receptor 4 signaling integrates intestinal inflammation with tumorigenesis: Lessons from the murine model of colitis-associated cancer. Cancers. 3: 31043113.

[22]. Hong, Y., Chao, W., Chen, M. and Lin B. (2009). Ethyl acetate extracts of alfalfa (Medicago sativa L.) sprouts inhibit lipopolysaccharide-induced inflammation in vitro and in vivo. Journal of Biomedical Science. 16: 64-75.

[23]. Honore P, Donnelly-Roberts D, Namovic M, Chong C, Wade C, Chandran P, et al. The antihyperalgesic activity of a selective P2X7 receptor antagonist, A-839977, is lost in IL-1alphabeta knockout mice. Behav Brain Res. 2009; 204(1):77-81.

[24]. Hsu, D., Chen, S., Chu, P. and Liu, M. (2013). Therapeutic effects of sesame oil on monosodium urate crystalinduced acute inflammatory response in rats. Springer Plus. 2: 659-668.

[25]. Jerca, L., Jerca, O., Mancaş, G., Constantinescu, I. and Lupuşoru, R. (2002). Mechanism of action and biochemical effects of nitric oxide (NO•). The Journal of Preventive Medicine. 10:

[26]. 35-45.

[27]. Kim, SF., Huri, DA and Snyder, SH. (2005). Inducible nitric oxide synthase binds, Snitrosylates, and activates cyclooxygenase-2 Heyne ex. Roth in rats. International Journal of Comprehensive Pharmacy. 1: 1-3.

[28]. Krishnaraja, AV., Rao, CBM, Sundararaja, D., Sengupta, K. and Trimurtulu, G. (2009). Anti-inflammatory activity of Vitex leucoxylon L. Bark extracts against Freund's complete adjuvant induced arthritis in Sprague Dawley rats. American Journal of Infectious Diseases. 5: 68-73.

[29]. Kumar, PP., Kumaravel, S. and Lalitha, C. (2010). Screening of antioxidant activity, total phenolics and GCMS study of Vitex negundo. African Journal of Biochemical Research. 47:191-195.

[30]. Kunwar, A. and Priyadarsini, KI. (2011). Free radicals, oxidative stress and importance of antioxidants in human health. Journal of Medical and Allied Sciences. 1: 53-56.

[31]. Lam, ANC., Demasi, M., James, MJ, Husband, AJ. And Walker, C. (2004). Effect of red clover isoflavones on Cox-2 Activity in murine and human monocyte/macrophage cells. Nutrition and Cancer. 49: 89-93.

[32]. Lawlor, G., Doran, PP., MacMathuna, P. and Murray, D. (2010). MYEOV (myeloma overexpressed gene) drives colon cancer cell migration and is regulated by PGE2. Journal of Experimental and Clinical Research. 29: 81.

[33]. Lee, S., Shin, S., Kim, H., Han, S., Kim, K., Kwon, J., Kwak, J., Lee, C., Ha, N., Yim, L. and

[34]. Kim, K. (2011). Anti-inflammatory function of arctiin by inhibiting COX-2 expression via NF- K $\beta$ pathways. Journal of Inflammation. 8: 16-24.

[35]. Lin, C., Crawford, DR., Lin, S., Hwang, J., Sebuyira, A, Meng, R., Westfall, JE, Tang, H., Lin, S., Yu, P., Davis, PJ. And Lin, H. (2011). Inducible COX-2 dependent apoptosis in human ovarian cancer cells. Carcinogenesis. 32: 19-26.

[36]. Lobo, V., Patil, A., Phatak, A. and Chandra, N. (2010). Free radicals, antioxidants and functional foods: Impact on human health. Pharmacognosy Reviews. 4: 118-126. 
[37]. Marnett, LJ. (2009). Mechanisms of cyclooxygenase-2 inhibition and cardiovascular side effects the plot thickens. Cancer Prevention Research. 2: 288-291.

[38]. Masoko P. and Eloff JN. (2007). Screening of twenty-four South African Combretum and six Terminalia species (Combretaceae) for antioxidant activities. African Journal of Traditional,

[39]. Complementary and Alternative Medicines. 4: 231-239.

[40]. McDaid, C., Maund, E., Rice, S., Wright, K., Jenkins, B. and Woolacott N. (2010). Paracetamol and selective and non-selective non-steroidal anti- inflammatory drugs (NSAIDs) for the reduction of morphine-related side effects after major surgery: a systematic review. Health Technology Assessment. 14: 1-180.

[41]. Milano, S., Arcoleo, F., Dieli, M., D'Agostino, R., D'Agostino, P., De Nucci, G. and Cillari, E. (1995). Prostaglandin E2 regulates inducible nitric oxide synthase in the murine macrophage cell line J774. Prostaglandins. 49: 105-15.

[42]. Moalem G and Tracey DJ. Immune and inflammatory mechanisms in neuropathic pain. Brain Res Rev. 2006; 51(2):240-64.

[43]. Mulabagal, V., Alexander-Lindo, RL., DeWitt, DL and Nair, MG. (2011). Health beneficial phenolic aldehyde in Antigonon leptopus tea. Evidence-based Complementary and Alternative Medicine. 10: 1093-1098.

[44]. Pala, FS., and Gurkan, H. (2008). The role of free radicals in ethiopathogenesis of diseases. Advances in Molecular Biology. 1: 1-9.

[45]. Park, G., Jun, J. and Kim, J. (2013). XH-14, a novel danshen methoxybenzo [b]furan derivative, exhibits antiinflammatory properties in lipopolysaccharide treated RAW 264.7 cells. Journal of Inflammation. 10: 1.

[46]. Patel, VR., Patel, PR., and Kajal, SS. (2010). Antioxidant activity of some selected medicinal plants in Western region of India. Advances in Biological Research. 4: 23-26.

[47]. Payne, R. (2000). Limitations of NSAIDs for pain management: Toxicity or lack of efficacy. Journal of Pain. 1: 14-18.

[48]. Pegg, RB. (2009). Characterization of the anti-inflammatory properties of Georgia-grown blackberries. (Rubus sp.) $1-5$.

[49]. Posadas, I., Rosa, S., Terencio, C., Paya, M. and Alcaraz, J. (2003). Cacospongionolide B suppresses the expression of inflammatory enzymes and tumour necrosis factor-a by inhibiting nuclear factor-kB activation. British Journal of Pharmacology. 138: 1571-1579.

[50]. Ricciotti, E. and Fitzgerald, GA. (2011). Prostaglandins and inflammation. Arteriosclerosis, Thrombosis, and Vascular Biology. 31: 986-1000.

[51]. Sakat, SS., Juvekar, AR. and Gambhire, MNJ. (2010). In vitro antioxidant and anti-inflammatory activity of methanol Oxalis corniculata Linn. International Journal of Pharmacy and Pharmaceutical Sciences. 2: 146-155.

[52]. Sales, KJ., and Jabbour, HN. (2003). Cyclooxygenase enzymes and prostaglandins in pathology of the endometrium. Reproduction. 126: 559-567.

[53]. Rao, AK. (2010). The tale of two COXs. Blood. 115: 921-922.

[54]. Tung, Y., Chua, M., Wang, S. and Chang, S. (2008). Anti-inflammation activities of essential oil and its constituents from indigenous cinnamon (Cinnamomum osmophloeum) twigs. Bioresource Technology. 99: 39083913.

[55]. Turini, ME., and DuBois, RN. (2002). Cyclooxygenase-2: A therapeutic target. Annual Review of Medicine. 53: $35-57$.

[56]. Vadivu, R. and Lakshmi, KS. (2011). In vitro and in vivo anti-inflammatory activity of leaves of Symplocos cochinchnensis (Lour) Moore ssp laurina. Bangladesh Journal of Pharmacology. 3: 121-124.

[57]. Wakefield, D. and Kumar, RK. (2001). Inflammation: Chronic. Encyclopedia of Life Sciences. Nature Publishing Group.

[58]. Wang, D. and DuBois, RN. (2006). Prostaglandins and cancer. Gut. 55: 115-122.

[59]. Wei XH, Zang Y, Wu CY, Xu JT, Xin WJ, Liu XG. Peri-sciatic administration of recombinant rat TNF-alpha induces mechanical allodynia via up regulation of TNF-alpha in dorsal root ganglia and in spinal dorsal horn: the role of NF-kappa B pathway. Exp Neurol. 2007; 205(2):471-84. 
[60]. Whitehead KJ, Smith CG, Delaney SA, Curnow SJ, Salmon M, Hughes JP, et al. Dynamic regulation of spinal pro-inflammatory cytokine release in rat in vivo following peripheral nerve injury. Brain Behav Immun. 2010; 24(4):569-76.

[61]. Wu, C., Lii, C., Liu, K., Chen, P. and Hseih, S. (2013). Anti-inflammatory activity of Gynura bicolor ether extract through inhibits nuclear factor kappa B activation. Journal of Traditional and Complementary Medicine. 3: $1-7$.

[62]. Yan, Y., Li, J., Ouyang, W., Ma Q., Hu, Y., Zhang, D., Ding, J., Qu, Q., Subbaramaiah, K. and Huang, C. (2006). NFAT3 is specifically required for TNF- $\alpha$-induced cyclooxygenase-2 (COX- 2) expression and transformation of Cl41 cells. Journal of Cell Science. 119: 2985-2995.

[63]. Zhuang, H., Pin, S., Li, X. and Doré, S. (2003). Regulation of heme oxygenase expression by cyclopentenone prostaglandins. Experimental Biology and Medicine. 228: 499-505.

[64]. Zhao, F., Wang, L. and Liu, K. (2009). In vitro anti-inflammatory effects of arctigenin, a lignan from Arctium lappa L., through inhibition on iNOS pathway. Journal of Ethnopharmacology. 122: 457-462. 